\title{
Est-on aux pizzas comme on est aux casseroles ? \\ Sur les emplois métonymiques des syntagmes prépositionnels en à avec un nom d'objet
}

\author{
Patricia C. Hernández \\ Instituto de Lingüística - Universidad de Buenos Aires \\ patrindez@yahoo.fr
}

\section{Introduction $^{1}$}

Les usages spatiaux de la préposition à évoquent des lieux non configurés où le repérage, loin d'être soumis à une analyse serrée, prend appui sur ce qui va de soi dans une situation donnée. Ainsi, dans "J'irai chercher Guy au train », peu importe que la rencontre ait lieu non pas dans le train mais sur le quai de la gare puisque l'assignation du lieu échappe à une topologie fixe : au train fonctionne comme « une sorte de rubrique associative, instanciée en fonction de l'expérience partagée par les interlocuteurs » (Cadiot, 1997b).

Le présent travail porte sur ces emplois locatifs jouant sur le savoir commun et focalise en particulier les séquences situatives avec des noms d'objet du type être à + article défini + nom d'entité de référence, par exemple Paul est aux casseroles. Notre objectif est d'explorer les rapports évoqués par ces syntagmes prépositionnels et de proposer une typologie des relations induites par les savoirs et les rituels associés aux entités en cause.

Après une brève description de l'état de l'art, nous aborderons, dans le cadre d'une sémantique cognitive, la dimension conceptuelle de ces rapports. Des occurrences attestées sur support électronique nous fourniront un éventail d'usages en contexte aptes à mettre en lumière la dimension pragmatique de ce type particulier de localisation. Seront évoqués la connaissance expérientielle des objets, leur corrélation avec des gestes et des actions prototypiques, le recouvrement des différents domaines convoqués par ce type de repérage ainsi que la nature métonymique des liens pragmatiques à l'œuvre dans le discours.

La typologie proposée à la fin de notre étude jette les bases d'une approche holiste du phénomène avec une prise en compte du fonds commun de connaissances sur lequel se tissent les interactions langagières.

\section{Caractérisation des rapports instanciés par la préposition à dans son emploi dit spatial}

\subsection{Abstraction, localisation et inférence}

De par son origine latine, la multiplicité de ses emplois et la commutation possible avec d'autres marqueurs, la préposition $\grave{a}$ est généralement considérée par grammairiens et linguistes comme étant abstraite, incolore ou vide (Brunot et Bruneau, 1956 [1933] ; Wartburg et Zumthor, 1973 [1947] ; SpangHanssen, 1963 ; Cadiot, 1997a). Cette abstraction se traduit, dans ses emplois spatiaux (répondant à la question Où est X?), par une caractérisation essentielle : l'a-descriptivité (Hernández, 2007, 2010). En effet, à la différence des prépositions considérées comme colorées ou sémantiquement pleines, ce relateur semble rendre compte d'une spatialité idéalisée, non fractionnée (Cadiot, 1997b : 200), sans détails contingents. 
La polarité abstraction-matérialité se traduit par une distinction entre localisation et configuration (Vandeloise, 1988). Par opposition à la configuration - opération de type descriptif, particularisante et nettement spatialisante, induite par des marqueurs plus colorés comme dans et sur - la localisation évoquée par la préposition à suppose un repérage a-descriptif qui situe une cible dont la position est inconnue ou imprécise par rapport à un site connu ${ }^{2}$ (Vandeloise, 1988: 126). Ainsi, dans Paul est à la gare, la gare s'avère apte à situer Paul sur la présomption d'un savoir commun (aussi bien sur le contexte d'énonciation que sur la réalité extralinguistique). C'est ce qu'illustre l'affinité entre la préposition $a ̀$ et les groupes nominaux introduits par l'article défini (Paul est à la gare) et, corrélativement, le refus de l'indétermination (*Paul est à une gare). La différence de comportement entre un marqueur configurationnel, par exemple dans, et le localisateur $\grave{a}$ est patente :

$$
\begin{aligned}
& \text { a. Paul est dans un / le / son lit } \\
& \text { b. Paul est au lit }
\end{aligned}
$$

L'énoncé (1a) constitue une description particulière empreinte de matérialité comme en témoigne la plasticité des combinaisons possibles - voire l'indétermination. Il met en scène la configuration d'une intériorisation, opposée, par exemple, à la superposition induite par Paul est sur le lit. Dans des énoncés de ce type, le terme lit fonctionne comme site d'une relation spatiale. En revanche, l'énoncé (1b) induit un repérage abstrait (a-descriptif) où le rapport locatif (réponse à la question Où est Paul ?) peut se doubler d'une indication situationnelle dans la mesure où l'entité 'lit' contribue à évoquer une position, une habitude, une situation (Franckel et Lebaud, 1992 : 101). Au point que être au lit est paraphrasable, selon les contextes, par des indications d'activité, état ou comportement général telles que, entre autres, dormir, se reposer, être alité. Ainsi le prouve d'ailleurs le fait que (1b) peut répondre non seulement à la question Où est Paul? mais aussi à Que fait Paul? voire Comment va Paul? Si, dans les emplois localisateurs, la connaissance partagée aide à l'identification, elle joue un rôle central dans l'interprétation de ces emplois, de nature plus notionnelle, sur la base de ce que Vandeloise appelle rituel ou routine sociale (1988 : 135, 1990 : 170), scénario habituel partagé par une communauté linguistique et culturelle.

Dans les distinctions vandeloisiennes entre configuration et localisation et, plus particulièrement, entre localisation et repérage selon une routine établie, transparaît la tension entre codage, pour les prépositions colorées, par exemple dans en emploi spatial, et inférence, pour les prépositions incolores comme à (Cadiot, 1997a). En effet le repérage construit par à active des inférences sur des interactions typiques associées conventionnellement au site. Ainsi, alors que des syntagmes comme dans la cuisine, semblent privilégier une indication de lieu - certes non exclusive - du type « La litière du chat est dans la cuisine ", les séquences à la cuisine semblent aptes à évoquer alternativement ou conjointement (i) des instructions interprétatives de localisation et (ii) une lecture notionnelle liée à un comportement, une

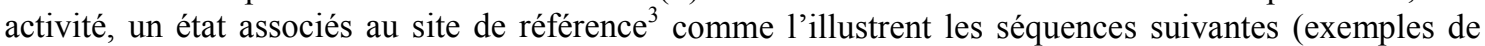
Katz, 2002) :

$$
\begin{aligned}
& \text { a. Où est Philippe? - Il est à la cuisine (et il y discute avec Catherine). } \\
& \text { b. Que fait Philippe? - Il est à la cuisine (il prépare le déjeuner). }
\end{aligned}
$$

Voyons des exemples attestés de localisation (3) et d'inférence d'activité pertinente, dans ce cas, cuisiner (4) :

(3) Afin de bien s'épanouir, votre eau de vie préférée doit éviter d'être remuée. C'est pourquoi un cellier muni d'un système anti-vibrations conservera de riches arômes. Pour bien l'utiliser, il est important de le placer dans un endroit plutôt calme. Par exemple, s'il est à la cuisine, on s'abstiendra d'installer cette dernière acquisition tout près d'un lave-vaisselle ou d'un réfrigérateur. (Celliers Ethier)

(4) Presto dispose d'un livre de recettes standardisé auquel les employés en cuisine ont accès à [sic] via un Ipad. En ayant accès constamment aux recettes et à une photographie couleur de la présentation des assiettes, ceux-ci seront autonomes plus rapidement et l'expérience de vos clients sera toujours à son meilleur, peu importe qui est à la cuisine. (Logiciel de gestion pour la restauration) 
En (3), à la cuisine renvoie à un endroit avec des caractéristiques précises activant une interprétation de simple localisation. En (4), à la cuisine construit un scénario d'activité - interprétation du type $s$ 'affairer à (Franckel et Lebaud, 1992 : 101) - où « peu importe qui est à la cuisine » serait paraphrasable par 'peu importe qui fait la cuisine / prépare les plats', etc. Bien entendu, l'on ne saurait attribuer à la seule préposition l'interprétation de l'énoncé en termes d'activité : la connaissance du caractère animé ou inanimé de la cible contribue fortement aux inférences. Ainsi, les lectures strictement localisatrices semblent fréquemment déclenchées par des énoncés mettant en scène des cibles inanimées (le cellier est à la cuisine) alors que les êtres vivants, dotés de la faculté d'agir (en (4) les employés en cuisine), apparaissent souvent comme cible dans des énoncés induisant une attitude, une manière d'être, l'effectuation d'une action associées au site. Encore une précision : les interactions entre cible et site ne sauraient être conçues comme 'données d'avance' : elles surgissent des transactions avec le contexte. En effet, bien que certaines des activités socialement instituées auxquelles le site se prête régulièrement soient plus saillantes (par exemple, cuisine $\rightarrow$ cuisiner), d'autres interactions pertinentes peuvent émerger du contexte selon les caractéristiques de la cible. C'est le cas de (5), par exemple, où l'interaction (manger) diffère de celle évoquée en (4).

(5) Mais où est passé doudou? Doudou, c'est un gourmand, un goulu, un glouton, un morfale...C'est sûr, il est à la cuisine! [...] Mais doudou n'a laissé derrière lui que des papiers vides et des miettes... (« Cailloux » Comptes, comptines et jeux de doigts)

Autrement dit, de même que d'autres unités linguistiques, la préposition à intervient dans un construction dynamique du sens comme déclencheur d'inférences en interaction avec son environnement co(n)textuel. Cette puissance inférentielle s'avèrera cruciale pour l'interprétation des syntagmes objet de notre étude. En emploi directionnel (Je vais au bureau) ou positionnel (Je suis au bureau), ce marqueur abstrait, localisateur et inférentiel, active un repérage a-descriptif qui, selon le contexte d'apparition, suscite les ajustements pragmatiques pertinents. Cette caractéristique n'a pas échappé aux linguistes qui, depuis une vingtaine d'années, ont produit un ensemble bibliographique de base sur les types de repérage induits par la préposition $\grave{a}$.

\subsection{Types de repérage}

Dans les lignes qui suivent, nous dresserons un état des lieux du traitement des relations évoquées par les emplois situatifs de la préposition.

\subsubsection{La notion de routine sociale}

Les travaux de Vandeloise sont éclairants sur cette question. Dans «Les usages spatiaux statiques de la préposition $\grave{a} »(1988)$, le linguiste décrit deux relations possibles :

- Localisation par un site connu ou fonction de localisation, repérage spatial à valeur référentielle : la préposition à localise par la coïncidence spatiale entre la cible et un site facilement identifiable. D'où la cooccurrence du marqueur avec les noms propres de lieu et les articles définis ${ }^{4}$ et sa relative incompatibilité avec les articles indéfinis.

$$
\begin{aligned}
& \text { (6) Léopold est à Liège } \\
& \text { (7) Élisabeth est à la plage } \\
& \text { (8) *Elisabeth est à une plage }
\end{aligned}
$$

- Evocation d'un rituel connu, repérage fonctionnel à valeur générique. En effet, considérant l'espace mis en mots dans le discours usuel en tant que lieu d'inscription du sujet en interaction avec son environnement dans un contexte donné, Vandeloise conçoit l'existence de relations fonctionnelles où le relateur évoque une routine d'interaction conventionnalisée. Ce repérage suppose l'existence d'un scénario habituel déclenché par un site avec une valeur socioculturelle suffisamment instituée : un lieu ou un objet. C'est ce que l'auteur appelle sites intégrés, liés à un contexte d'énonciation mais aussi à une routine sociale générant un processus de conventionnalisation linguistique. 
Vandeloise (1988) dégage trois caractéristiques principales de l'emploi de à avec ces sites particuliers :

(i) comportement conforme aux règles imposées par une routine sociale associée au site sans marquage topologique particulier du lieu physique. Ainsi, l'énoncé Baudouin est au piano évoque pour l'actant une place compatible avec la pratique du piano : on l'imagine assis sur un tabouret, tourné vers le clavier ;

(ii) exclusion des cibles non pertinentes pour la routine évoquée par le site : l'acceptabilité de la cible dépend de sa capacité à s'intégrer dans un rituel évoqué par le site. En effet, supposant une interaction typique, le scénario lié au site, culturellement enraciné, pose des contraintes de pertinence comme en témoigne la différence d'acceptabilité entre L'enfant est à l'école, ? Le chien est à l'école, ?? L'arbre est à l'école ;

(iii) caractère générique: les sites intégrés n'admettent pas de qualifications propres à un membre particulier de la catégorie. C'est ce qu'illustrent les énoncés Baudouin est à la caserne, * Baudouin est à la caserne dont les barbelés brillent sous la pluie.

\subsubsection{Le sens télique}

La distinction vandeloisienne entre fonction de localisation et site intégré est reprise par Borillo (2001) qui identifie trois cas de figure :

- Un sens spatial référentiel, correspondant peu ou prou à la localisation par un site connu (qui répond naturellement à la question $O \dot{u}$ ?), par exemple, être à la cave, au salon, au château.

Borillo relève les restrictions qui pèsent sur l'emploi des noms d'objets matériels comme repère localisateur ( ? Paul est à la chaise). En effet, acceptable pour les itinéraires (Paul est [arrivé] au rocher) ou comme forme condensée pour des indications de rangement habituel (La clé est [pendue] au clou), la préposition $\grave{a}$, affirme l'auteur (2001:88), est rétive, dans sa fonction de localisation, aux combinaisons avec des noms d'objet.

- Un sens fonctionnel télique - selon le rôle télique de la Structure de Qualia (Pustejovsky, 1995) - où le sens spatial est mis en sourdine et cède le pas à une interprétation en termes d'activité, comportement ou état induits de manière prototypique par le site de référence. Ce sens rejoint la caractérisation des sites intégrés de Vandeloise. Par exemple, être au placard, au trou, au ciel, à la rue, au tapis.

- Des cas de double interprétation, comme être à l'école, au lit, au café, au piano, à la poubelle, au cimetière, aux champs. Étroitement dépendantes du contexte, ces expressions donnent lieu à des lectures tantôt spatiales tantôt téliques, comme l'illustre le cas de être à l'école employé d'abord avec un sens spatial et ensuite avec un sens télique :

Les enfants étaient à l'école et le village, désert.

(13) C'était le temps heureux où il était à l'école.

Remarquons que, dans cet exemple, l'alternance sémantique tient non seulement aux deux types de rapport susceptibles d'être évoqués par la préposition à mais aussi au fait que le lexème école englobe diverses facettes (Cruse, 1986) telles que [IMMEUBLE], [INSTITUTION], [PERSONNEL], [ENSEIGNEMENT], par exemple. Un éclairage particulier de la facette [IMMEUBLE] du nom régime en interaction avec la préposition peut susciter une interprétation plus localisatrice ; la saillance d'une facette plus abstraite, par exemple [INSTITUTION], peut contribuer à une lecture plus notionnelle, distinction qui apparaît dans les exemples de Corblin (2011) : «L'école du village a été repeinte en blanc » (lieu), «L'école du village donne aux enfants une excellente formation » (institution sociale). Entre les différentes facettes, un continuum qui se ne se prête pas toujours à une discrétisation, surtout dans le cas des cibles animées accompagnées régulièrement d'une présomption d'interaction avec l'environnement. Ici, même en (12), à 
l'école active une lecture en termes d'interaction typique et pertinente - à preuve, les doutes par rapport à des formulations du type : ? Les chiens étaient à l'école ${ }^{5}$. Nous reviendrons sur ce point dans la section 3.2 .

Dans la conclusion de son article, Borillo signale la coexistence des deux interprétations et note une tendance généralisée à privilégier le sens télique autant pour les constructions avec les noms de lieu que pour celles comportant des noms d'entités matérielles; tendance qui semble confirmée par la fixation de la préposition avec des noms abstraits représentant des actions ou des processus (être au spectacle, au travail, au repos, au lavage, au rebut) au point d'induire des correspondances du type : être au bureau $\rightarrow$ être au travail, être à la poubelle $\rightarrow$ être au rebut (cf. aussi note 3 )

\subsubsection{La distinction entre lieux et objets}

Dans divers travaux, Aurnague $(2004,2009,2010)$ définit deux emplois de la préposition à sur la base d'une différenciation entre lieux et objets. Suivant la distinction vandeloisienne entre fonction de localisation et sites intégrés, le linguiste identifie :

- Un emploi statique localisateur où la préposition à est suivie d'un nom de lieu, le lieu étant caractérisé comme une entité matérielle fixe ou stable dans un cadre de référence donné qui définit une 'portion d'espace'. Tel est le cas des lieux géographiques identifiés par des noms propres ou des noms communs de lieux géographiques introduits par l'article défini. L'emploi localisateur de la préposition est donc lié à l'indication de lieux spécifiés (2009: 36).

- Un emploi non localisateur interprétable en termes d'activité en cooccurrence avec des objets i.e. des entités qui ne satisfont pas aux critères énoncés pour les lieux, tel le cas d'un couteau, un balai, un verre, une commode, un poteau ou un rocher. Ces emplois, même en cas de position spécifiée, ne sont pas considérés comme pouvant localiser la cible (*Léopold est au rocher, * La mouche est au verre). En revanche, intégrés à des 'routines sociales' (Vandeloise, 1990), ils déclenchent des interprétations en termes d'activité où la cible peut jouer un rôle d'agent (être au piano) ou de patient (être au congélateur) (Aurnague, 2010).

Un cas particulier serait celui des bâtiments ou habitations, considérés par Aurnague (2009: 36) comme des entités mixtes ayant des propriétés communes avec les deux classes établies : stabilité et détermination d'une portion d'espace, comme les lieux géographiques, et structuration en 'composants' ou parties fonctionnelles, comme les objets.

L'auteur signale que non seulement la classe des objets est susceptible de recatégorisation en lieu selon le contexte d'emploi ${ }^{6}$ mais aussi et surtout que les usages localisateurs et non localisateurs ne sont pas forcément disjoints.

Suivant Aurnague, Corblin (2011) distingue, lui aussi, lieux et objets et associe ces deux catégories à l'évocation d'une activité, lien qu'il désigne, reprenant Borillo (2001), comme interprétation télique. Deux cas sont évoqués dans «Locus et telos : aller à l'école, être à la plage » (Corblin, 2011) :

- Avec des noms de lieu : Les syntagmes prépositionnels du type $\grave{a}+$ article défini + nom de lieu (à la plage) déclenchent, affirme le linguiste, deux lectures possibles : une construction locative, simple indication spatiale (Le taxi est à la plage) et une construction télique, évocation d'une activité (Pierre est à la plage). Dans le cas des cibles animées situées par rapport à un lieu, locus et telos peuvent se superposer. C'est ce qu'il désigne comme 'activités-lieu'.

- Avec des noms d'objet (Marie est au piano, La dinde est au four), Corblin écarte la possibilité d'un repérage spatial ( $*$ La mouche est au piano) et privilégie l'interprétation télique ${ }^{7}$ où, de manière générale, à localise par l'implication de l'activité à laquelle est destiné l'objet en question ('activités-objet'). Il s'agit, pour la plupart, d'artefacts suscitant des interprétations téliques, classées par l'auteur en agentives pour les humains (le céramiste était au four) et en objet affecté pour les objets (la sculpture était au four). 
Le linguiste s'interroge au sujet du référent dénoté par les noms d'objet (objet concret ou activité associée ?) pour conclure que l'interprétation télique n'implique pas un glissement référentiel mais un 'forçage' (coercition de type (type coercion) selon Pustejovsky, 1995). D'où l'application non systématique du mécanisme, qui affiche peu d'affinité avec certains lexèmes. Corblin évoque le cas de certains outils ( ? Pierre est au marteau / tournevis / pinceau), de certains instruments de musique (à la différence de être au piano, l'acceptabilité de être à la guitare / à la flûte à bec semble restreinte au contexte des formations musicales), des parties d'un véhicule (Pierre était au volant, ? son épouse au siège passager, ses enfants à la banquette arrière, et ses bagages au coffre). Autant de cas qui rendent compte d'un comportement non uniforme, l'acceptabilité des énoncés dépendant souvent de leur insertion dans un scénario d'activité. En effet, n'étant pas un mécanisme primitif de la grammaire, conclut l'auteur, ce phénomène aléatoire ne touche que certains noms d'objet.

C'est ce domaine mouvant des séquences décantées par l'emploi en discours que nous nous proposons d'étudier. Nous porterons notre regard sur les formulations où le verbe être est suivi de syntagmes prépositionnels évoquant des comportements, activités ou états. Trouveront leur place dans notre étude des lexèmes désignant aussi bien des lieux que des entités considérées comme étant des 'objets', les deux types d'entités étant traités selon les appréhensions perceptives et les praxis qui leur sont associées.

\section{Le cas des emplois liés à des scénarios établis}

Dans les sections qui suivent, nous présentons, une approche du phénomène qui, à l'aide de certains concepts fondateurs de la sémantique cognitive, éclaire d'un jour nouveau l'analyse des séquences où la préposition $\grave{a}$ introduit des syntagmes non seulement avec des noms de lieu mais aussi (et surtout) avec des noms d'objet. Pour cela, il nous semble indispensable d'intégrer, d'une part, la prise en compte de la dimension praxéologique des lieux et des objets et, d'autre part, l'observation des comportements linguistiques effectifs.

En effet, une approche non insulaire du langage conçu en rapport avec d'autres capacités cognitives comme la perception visuelle et le système moteur (Jackendoff, 1983 ; Langacker, 1987) permettra de mieux saisir les modes de constitution des entités qui nous entourent, les aspects privilégiés par la mise en mots de ces entités ainsi que leur progressive conventionnalisation en langue.

Quant aux énoncés analysés, notre exposé vise à faire émerger des productions attestées qui mettent en lumière les fonctions pragmatiques sous-tendant certaines formulations souvent absentes des études linguistiques. Ces occurrences ont été recueillies sur Google France le 25 octobre 2011. Un corpus complémentaire a été relevé sur le même moteur de recherche le 3 mars 2012. Même si nous n'en offrons pas ici une analyse quantitative, il faut souligner que les énoncés retenus correspondent, pour la plupart, à des séquences moyennement fréquentes et jugées acceptables par des informateurs.

Sur ces prémisses, abordons, pour commencer, le caractère expérientiel des objets.

\subsection{La corrélation entre objets et actions}

Aussi bien la Gestalttheorie que, plus tard, la sémantique cognitive accordent une place de choix à l'acte d'utilisation (même esquissé ou virtuel) comme mode de (re)connaissance des objets. Ainsi, écrit Janet dans Les débuts de l'intelligence (1935:41) :

\footnotetext{
Quand nous percevons un objet, un fauteuil, par exemple, nous disons qu'en le voyant nous savons ce qu'est cet objet, que nous le reconnaissons, mais nous croyons ne pas faire d'action à ce moment, car nous restons debout, immobiles, en percevant le fauteuil. Il y a là une illusion, en réalité nous avons déjà en nous l'acte caractéristique du fauteuil, ce que nous avons appelé le schéma perceptif, ici l'acte de nous asseoir d'une manière particulière dans ce fauteuil.
}

Un rapport analogue s'établit entre la nomination des objets du niveau de base (des termes tels que fleur, ballon, chat, etc.) et les gestes typiques qui y sont associés. Ainsi, affirme Brown (1965: 318), des 
désignations telles que fleur, ballon, chat demeurent mentalement connectées à des actions telles que sentir, faire rebondir ou caresser. A tel point, renchérit-il, qu'elles peuvent fonctionner comme des symboles et évoquer des entités sans équivoque dans un jeu de charades.

C'est que le type de rapport perceptuel ou forme spécifique de contact que l'on entretient avec ces entités s'avère être un élément constitutif de la nomination et, partant, du comportement des lexèmes en discours. Sur ce point, une telle conception n'est pas éloignée de la praxématique telle qu'elle est définie dans les travaux de Siblot (1997) et garde des points de contact avec la théorie des propriétés extrinsèques du référent postulée par Cadiot et Nemo (1997a, 1997b). Ainsi, plutôt que de concevoir les mots comme des descriptions d'objets, il faut y voir, soulignent Cadiot et Nemo, « des clés d'accès, ou encore des routines, des évocations, des synthèses de l'expérience (variablement associée à tel ou tel 'référent') (1997b : 129).

D'où il s'ensuit que la fusion entre objets et rapport expérientiel - 'objets activités' pour Corblin (2010) ne représente pas un cas particulier mais, au contraire, le mode naturel d'appréhension de l'univers environnant. Et ce rapport expérientiel est mis en scène dans des frames (Fillmore, 1982), cadres d'interactions typiques supposant des rôles et des comportements selon notre connaissance du monde. Cette connaissance est organisée dans des modèles souples et évolutifs désignés par Lakoff (1987) comme des Modèles Cognitifs Idéalisés fournissant le matériau nécessaire à des connexions de nature métonymique - nous reviendrons sur ce point en 3.3 -. Ce catalogue de situations est donc constamment formé / enrichi / modifié / ajusté selon notre environnement social et culturel au fil du temps et du discours. Les situations associées aux objets - de même qu'aux lexèmes correspondants - façonnent les représentations de ces entités et interviennent dans la construction du sens.

Ainsi, tout un univers de création artistique, y compris des gestes caractéristiques, semble évoqué par les énoncés suivants ${ }^{8}$ où les syntagmes prépositionnels avec des termes tels que pinceaux, toiles, crayons renvoient à l'exécution d'une peinture ou d'un dessin. En (14-17), des parallélismes tels que être aux pinceaux / manier la plume en (14) ou des corrélations entre être aux pinceaux et aux toiles / se lancer dans un nouvelle création en (15) mettent en évidence le caractère praxéologique de ce repérage : cet emploi de à n'a pas pour fonction première de localiser - en (17) la cible (R. Di Martino) n'est vraisemblablement pas 'aux crayons' au moment de l'énonciation - mais d'évoquer une activité, un procès, un mode d'interaction associé à l'objet désigné, dans sa matérialité ou dans sa dimension mythique (16).

(14) Nous sommes deux dans cette entreprise : l'ami Théophane, Béninois, est aux pinceaux, pendant que moi, un Blanc, Français, manie la plume.

Tenaillé par sa créativité, l'homme est aux pinceaux et aux toiles dès son retour du travail. Il se lance chaque fois dans une nouvelle création.

Cette fois-ci, je voulais avoir l'avis d'un créatif, le gars qui est aux pinceaux (pinceaux virtuels de nos jours, évidemment)...

C'est Richard Di Martino, déjà dessinateur de la série Malek Sliman chez Vents d'Ouest qui est aux crayons et apporte une esthétique résolument moderne à la série.

Il en va de même pour l'évocation de la routine d'organisation d'un spectacle ou la préparation d'une soirée : plus que les entités dénotées, ce sont les gestes propres à chaque fonction qui sont évoqués par les syntagmes prépositionnels en (18-22). L'interprétation en termes d'activité associée aux entités des domaines dénotés est manifeste : un lien s'établit entre le potentiel évocateur d'entités matérielles telles que les lumières ou les éclairages et l'indication d'une activité ou d'une fonction, la direction artistique en (18) ou la scénographie en (19), les SP en à étant souvent paraphrasables par la désignation d'activités ou de procès tels que la conception / réalisation / production / confection, etc. (cf. aussi 3.3.3). Ces séquences en $\grave{a}+$ nom d'objet peuvent également être coordonnées à des verbes, par exemple, en (21), préparer, servir, négocier. Enfin, il convient de signaler (nous reviendrons sur cela en 3.2 et 3.3.2) que les SP de ce type se trouvent souvent dans des contextes de distribution de rôles (18-22) : 
Brian Smith signe la scénographie, Normand Thériault est aux costumes, Guillaume Houët aux éclairages et Claude Naubert à l'environnement sonore.

$$
\text { Jean-Philippe Bourdon est aux lumières. Johnny a déjà débuté ses répétitions. }
$$

(21) José est au bar pour préparer les cocktails, Olivier sert les knacks, Yvon négocie avec les voisins, Alfonso est aux lumières.

L'un est au clavier quand l'autre est aux platines...

Le degré de saillance perceptuelle et culturelle de certaines entités, leur fréquence d'apparition dans certaines situations tend à cristalliser des séquences qui, convoquées plus souvent, verront leur ancrage cognitif se traduire par une fixation linguistique. Les critères d'un tel ancrage dépendent, eux, des pratiques des sociétés tant du point de vue extralinguistique (saillance de certains objets) qu'au niveau linguistique (prégnance de certains termes et donc stabilisation de certaines séquences parmi toutes les combinaisons possibles). Fruit d'un socle d'expériences partagées et non pas d'un calcul, ces combinaisons sont difficilement prédictibles. Nous rejoignons sur ce point l'avis de Corblin (2011).

Ainsi si le fourneau et la cuisinière servent à cuire des aliments, ce sont les formulations être au fourneau / aux fourneaux ${ }^{9}$ et non pas être à la cuisinière / aux cuisinières qui apparaissent le plus fréquemment pour évoquer la routine de la préparation culinaire.

Madame qui est au fourneau cuisine admirablement bien, avec de bons produits bios.

La dame est aux fourneaux du matin au soir pour mitonner ses recettes...

\title{
3.2 L’inséparabilité des repérages
}

Comme il a été signalé en 2.2.2, qu'il s'agisse d'un lieu ou d'un objet, la routine sociale associée au site est apte à déclencher l'inférence d'une interaction typique avec la cible. En effet, liés à des scénarios établis, les emplois étudiés tiennent à un rapport expérientiel travaillé et retravaillé par la pratique quotidienne - au-delà des classements strictement ontologiques. Ce mode de donation de l'univers environnant s'avère, nous semble-t-il, rétif aux cloisonnements du point de vue pragmatique (nous avons effleuré ce sujet en 2.2.2). En effet, le frame (Fillmore, 1982 : 111), en tant qu'interaction typique définie par la culture, les institutions, l'expérience, suppose une vision intégrée :

\begin{abstract}
By the term 'frame' I have in mind any system of concepts related in such a way that to understand any one of them you have to understand the whole structure in which it fits; when one of the things in such a structure is introduced into a text, or into a conversation, all of the others are automatically made available.
\end{abstract}

Cette vision holiste suppose l'évocation de rôles et d'interactions mettant en jeu globalement des objets et des lieux liés dans une routine établie. Qui plus est, certains objets associés à une activité typique sont mentalement placés dans un lieu typique, toile de fond de la scène d'interaction. Ainsi, des ustensiles tels que les casseroles semblent se détacher sur le décor d'une cuisine et non pas - sauf exception - dans le salon ou la chambre à coucher, lieux qui semblent abriter naturellement, selon le cas, des objets tels que qu'un fauteuil, un tableau, un lit ou un réveille-matin. Ces éléments (lieux, par exemple, la cuisine, la chambre à coucher, et objets, par exemple des casseroles, un lit) se trouvent intégrés au frame correspondant à l'activité en question, par exemple 'faire la cuisine' ou 'dormir'. Leur saillance cognitive les rend aptes à évoquer l'action ${ }^{10}$.

Dans la mesure où, dans notre appréhension du monde, nous construisons des synthèses d'expérience sur la base autant des objets familiers (cf. 3.1) que des lieux d'interaction - nous l'avons signalé, les lieux sont aptes à déclencher des inférences de comportement -, les deux catégories sont convoquées à égalité ou activées de manière solidaire pour la construction du sens. C'est ce qu'illustrent les exemples suivants où le syntagme prépositionnel avec le nom de lieu (cuisine) ou ceux avec des noms d'objets (marmites, casseroles et couscoussières) renvoient au domaine des préparations culinaires et de la gastronomie : 
Restaurant indien Le Maharaja, Paris. Le père est à la cuisine avec l'expérience de la cuisine indienne et sri lankais [sic] traditionnelles depuis toujours, la mère dans la salle, à l'écoute de chacun...

(26) Depuis 1961, la famille Aïssa est aux casseroles et aux couscoussières. La propriétaire actuelle est la belle-fille du fondateur...

(27) Rien de tel qu'un bon réveillon pour passer d'une année à l'autre surtout lorsque votre cuisinier préféré est aux marmites.

A cela s'ajoute un phénomène d'extension: aussi bien pour les objets que pour les lieux, dans bon nombre de cas, le rapprochement entre la fonction du site (lieu ou objet, par exemple la cuisine ${ }^{11}$ ou les fourneaux) et l'activité de la cible (cuisiner) déclenche des inférences sur le rôle de la cible (cuisinier/ière). Les cibles animées se voient ainsi attribuer des rôles selon les lieux / objets dénotés comme site de l'interaction. Dans les énoncés ci-dessous, l'interprétation en termes d'activité, voire en termes de rôle semble activée autant par le nom de lieu (cuisine) que par les noms d'objets (fourneau, casseroles).

C'est aussi une affaire de famille où le fils est à la cuisine et le père en salle.

(29) Le gérant Giuseppe est au fourneau et vous prépare les meilleurs plats de son pays alors que sa femme Rosa se plie en quatre pour vous servir en salle.

(30) L'équipe de travail est recrutée au sein même de la famille. La maman est aux casseroles avec mari et fils comme aides de cuisine.

(31) Georges est aux casseroles et fonctionne comme cuistot de service.

La fusion entre la fonction du site (lieu /objet) et le rôle de la cible génère, comme effet résultatif, une certaine spatialisation du rôle / métier. Ainsi en témoignent les organigrammes des institutions, par exemple, ou les expressions courantes dans le discours pour l'indication d'une profession, telles que «Il est où, Paul, chez Total ? » «Il est au marketing / à la comptabilité / à la facturation / au secrétariat ». Et même «Il est où, Pierre, chez Carrefour?» «Il est aux produits frais / aux fruits et légumes / aux liquides / aux alcools » avec des degrés de spécificité variables (cf. aussi 3.3.1). C'est le cas des énoncés suivants :

\section{Dans quels rayons vous avez bossé ? Moi ça fait un an que je suis aux liquides.}

Depuis un an, je suis aux assiettes, alors que mes collègues changent de poste tous les trois mois. (Chez un traiteur)

La spatialisation découlant du lien entre lieux / objets et rôles dans un même modèle cognitif apparaît nettement dans les énoncés suivants où le terme place ${ }^{12}$ spatialise une répartition des fonctions et des sphères d'influence dans un ordre social établi :

La place des femmes est à la cuisine. C'est leur monde.

La fureur des chefs réformistes qui clamaient que la place des femmes est aux casseroles, elles ne doivent pas s'occuper de politique...

Avoir accès à tous les postes, quels qu'ils soient. Mais surtout, ne pas se laisser influencer par les farces de mauvais goût comme " ta place, c'est aux chaudrons ». (Site québécois)

On le voit, dans certains cas, plus qu'une simple localisation spatiale, les syntagmes en $\grave{a}+$ article défini + nom de lieu (cuisine) ou d'objet (casseroles / chaudrons) fournissent un repérage sur la base d'une activité typique induisant un rôle, lui, spatialisé et dans sa conceptualisation et dans sa mise en mots. Et la spatialisation n'est jamais anodine : le caractère générique (les femmes) ainsi que les deux activités mises en parallèle en (35), 'être aux casseroles' / 's'occuper de politique', semblent dessiner un espace symbolique hiérarchisé, objet d'investissements complexes. Il va de soi que cette 'localisation' métaphorique traduit plus des représentations sociales qu'elle ne décrit de localisation physique.

Autre exemple : 
Ici, la spatialisation joue encore pour une certaine ghettoïsation des activités. Le lien est évident entre les éléments co-présents travail et être aux poubelles et à la plonge désignant l'activité, le rôle. Notons que, ici, l'inférence d'activité (définissant un rôle) est suscitée autant par un nom dénotant un objet (poubelles) que par un lexème (plonge) susceptible de désigner une activité ou un lieu ${ }^{13}$.

Reste à définir la nature des associations évoquées. Dans la prochaine section, nous abordons les connexions pragmatiques à l'œuvre dans ce genre de formulation et proposons un essai de typification pour les emplois étudiés.

\subsection{Liens pragmatiques et accès mental à un modèle cognitif}

Nous formulons l'hypothèse que, dans la conceptualisation des relations - et l'expression de ces rapports à l'aide de syntagmes prépositionnels - l'être humain a recours à des connexions de type métonymique. Dépassant l'approche rhétorique classique qui conçoit la métonymie comme une affaire de mots, la sémantique cognitive y voit un phénomène conceptuel : il s'agit d'un processus dans lequel une entité conceptuelle fournit un accès mental à une autre entité dans un même Modèle Cognitif Idéalisé (Radden \& Kövecses, 1999 : 21). En effet, la tradition culturelle par rapport aux objets, habitudes, comportements, permet des connexions dans un réseau conceptuel complexe; ces connexions exploitent des relations prototypiques ou idéalisées constituant un pont mental entre des lieux, des objets, des événements, des situations en vertu de leur proximité conceptuelle selon la saillance de l'entité source, par exemple les casseroles pour l'action de cuisiner. Ainsi, des connexions métonymiques sont â l'œuvre dans des processus inférentiels spécialement convoqués par l'emploi de la préposition à. Dans cette perspective, plus d'une connexion peut être établie entre les entités convoquées en discours ce qui impose, nous semble-t-il, une modélisation plus souple que celle de l'attribution généralisée d'un rôle télique aux objets dénotés par la tête du syntagme prépositionnel.

Selon un continuum entre espace, temps et notion - avec d'éventuels recouvrements -, nous proposons une taxinomie ouverte aux inférences contextuelles (cf. 3.3.5) qui prévoit, à titre provisoire, cinq types de rapports métonymiques pour les séquences être $\grave{a}+$ article défini + nom d'objet. En présentant ce modèle souple et continu, nous visons, très concrètement, à dépasser une discrétisation entre lieux et objets et à étendre la modélisation des emplois avec des noms d'objet au-delà du rôle télique des artefacts.

\subsubsection{Objet $\rightarrow$ Lieu}

Il faut signaler l'existence d'une connexion métonymique par contiguïté cognitive entre objets et lieux ${ }^{14}$, avec une visée localisatrice et, le cas échéant, inférence situationnelle et comportementale. C'est, dans les exemples qui suivent, la métonymie 'le produit pour le lieu', par exemple « les surgelés » pour «le rayon des produits surgelés». Usuel dans le discours, ce type de repérage est particulièrement manifeste dans l'organisation spatiale des grandes surfaces ou des grands magasins :

(38) Un hypermarché aux rayons complets - alimentation, bijouterie, vêtements, très grand choix mais il ne faut pas avoir oublié le pain quand on est aux alcools à moins de chausser des rollers...

(39) L'Indien est aux surgelés et la dame aux chaussures aux céréales, tout cela a forcément un sens.

(40) Mes parents sont aux vêtements et comme je cherche un jeu très précis pour ma console et qu'ils n'y comprennent rien entre un game-cube et un PC., j'achète moi-même, mais c'est eux qui paient.

(41) Je suis partie, si on me cherche je suis au rayon pizza surgelées [sic] de Carref*** heu non tout compte fait tant qu'à y être je suis aux glaces... 


\subsubsection{Objet $\rightarrow$ Activité liée à la fonction}

Une connexion métonymique entre objets et activités s'établit selon la fonction de l'entité en question, entité qui, servant à exécuter, devient un instrument (prototypiquement un inanimé utilisé pour réaliser une action). Les cas les plus représentatifs se présentent avec des instruments (de musique), outils et ustensiles. Ce type de connexion recoupe l'interprétation télique proposée par Borillo (2001) et Corblin (2011) : l'entité nommée évoque l'action typique à laquelle l'objet est destiné. C'est le cas, par exemple, des casseroles et des marmites pour l'action de cuisiner où la connaissance encyclopédique et le frame correspondant à chaque situation orientent l'inférence d'activité. Il faut remarquer que, dans certains cas (par exemple, 45-47), l'emploi de à de type routine sociale s'accompagne d'une répartition des activités / tâches dans le cadre d'un scénario prédéfini (cf. aussi exemples 14, 18-22).

Abdou est aux casseroles, ou plutôt à la casserole ${ }^{15}$, puisqu'il n'y en a qu'une. Lui créé [sic] de la bouffe pour quinze personnes...

Afin de nourrir les petits colons, Philippe est aux marmites et aux casseroles. Celui qui sert habituellement les élèves du lycée d'Artois s'est entouré d'un bataillon féminin pour l'aider dans sa tâche.

La répartition traditionnelle des rôles est d'ailleurs en vigueur chez les Blaser : c'est Beni qui est au gril, son épouse Cora s'occupant de tout le reste.

Depuis les premières éditions de Mom'en scène, ils sont fidèles à leur mission "petits déjeuners ». Pendant qu'Enzo, le président, est aux cafetières, Lucien fait chauffer le lait au piano, Simone, Maria et Christiane disposent les bols au réfectoire et dispatchent les jus de fruits et autre produits laitiers sur les consoles. Toute l'équipe se souvenait de nous et jusqu'au cuistot qui est au wok...

Dans ce groupe, on peut inclure les noms désignant des instruments de musique, généralement cités comme déclencheurs de postures, gestes, activités prototypiques. Il faut remarquer le potentiel évocateur de ces formulations métonymiques non seulement en termes d'activité mais aussi en tant qu'indicateurs d'une spatialisation des rôles. Le cas d'un orchestre s'avère paradigmatique pour la distribution de rôles et l'organisation d'un groupe.

Cornelia est à la Clarinette, Galabrina à la Viole, Marnix au Violoncelle et Daniel, Mark et Maike au violon. Kurt dirige tous ses artistes de main de maître.

(49) Céline est au saxophone, accompagnée par ses deux compères à la basse électrique et à la batterie.

Ces contextes d'emploi ont des caractéristiques spécifiques. Comme le signale Corblin (2011) (cf. 2.2.3), à la différence de être au piano, certaines séquences du type être à la flûte à bec, affichent un degré d'acceptabilité variable selon qu'elles se trouvent dans un contexte de formation musicale où elles semblent naturelles ou dans un cadre quotidien où leur acceptabilité diminue. Nous citons un exemple proposé par un relecteur anonyme : « Dans le salon, Paul était à la table de travail et Marie au piano / ?? à la flûte ». Voilà qui permettrait de distinguer deux cas de figure pour l'association Objet $\rightarrow$ activité :

(i) le cas des routines sociales au sens large, dont la fixation linguistique s'avère irrégulière et partant difficilement prédictible selon les propriétés ontologiques des objets, ce qui explique la différence d'acceptabilité entre (50) et (51) :

\section{Paul est au piano}

$$
\text { ?? Jean-Pierre est à la flûte }
$$

(ii) le cas des routines instituées évoquant une fonction établie d'avance dans un ensemble consacré à une activité typique. Ce type de scénario (par exemple pour les formations musicales) facilite l'acceptabilité de certaines séquences douteuses prises isolément :

\section{Paul est au piano, Jean-Pierre à la flûte, Isaac au violon}




\subsubsection{Objet $\rightarrow$ Activité liée à la production}

Il s'agit d'une connexion métonymique entre objets et conditions d'apparition, de production des entités correspondant au rôle agentif du Lexique génératif de Pustejovsky (1995). Être à suivi de la désignation d'un objet spécifié renvoie à la conception, confection, préparation, débit des entités nommées. Dans ces énoncés, la cible (prototypiquement un sujet animé qui initie intentionnellement l'action) est montrée comme étant la source de l'objet site.

C'est pas Ronald Mac Donald qui est aux sandwichs, sinon bonne digestion!

(54) Sylvain règne sur une salle qui a beaucoup de succès, le Rock Ambiance, on y danse sur les Blues Brothers, les Breuvachons et Matmatah. Thierry n'est pas le moins sollicité, il est aux pizzas, hot dog et crêpes sur les terrasses extérieures. Pour étancher sa soif cinq bars sont disponibles.

Le patron prépare les plats, la patronne est aux pizzas, le service est assuré par une serveuse souriante et efficace.

(56) Une production fastueuse, Christian Lacroix est aux costumes.

D'autres exemples de ce type de connexion ont été présentés dans la section 3.1 où, par exemple, être aux costumes se trouve inséré dans un ensemble d'activités liées à la préparation d'un spectacle et associé à l'activité de création (mise en relief par le concept de 'signature' d'un auteur) : (19) «Brian Smith signe la scénographie, Norman Thériault est aux costumes... ».

\subsubsection{Objet $\rightarrow$ Situation / évolution}

Avec certains ajustements cotextuels, une connexion métonymique au niveau conceptuel et expérientiel met en rapport objets et indication situationnelle voire indication d'un stade dans une évolution. En effet, en interaction avec leur contexte, certains SP construisent un repérage de nature qualitative où la cible est identifiée par son rapport à des objets, rapport qui ne semble pas susceptible d'interprétation instrumentale ni d'identification agentive. Reposant sur l'inférence d'une interaction pertinente entre cible et site, il s'agit, dans de nombreux cas, d'une conduite de consommation (selon un libre choix ou sous certaines contraintes), paraphrasable, selon les domaines, par utiliser, acheter, manger, boire, porter, mettre, etc. Cela dit, il va de soi qu'il ne s'agit pas de formulations équivalentes : l'emploi du verbe être avec ses résonances existentielles et prédicationnelles dépasse la simple indication d'action. A titre d'exemple, «Je suis aux piles rechargeables » (57) engage l'individu, le situe, le catégorise plus que ne le ferait «J'achète / utilise des piles rechargeables ». De même, des énoncés tels que " mon pauv'mari est aux pizzas » (64) ou « une partie [...] de la population est aux sandwichs » (60) construisent des repérages situationnels engageant l'individu où le SP en interaction avec le co(n)texte évoque une contrainte (être réduit à) de manière plus connotée ${ }^{16}$ que «mon pauv'mari mange des pizzas » ou «la population mange des sandwichs ». Voici des exemples d'emploi :

(57) Je suis aux piles rechargeables! Moi aussi je suis pour l'environnement ! (Site Tippmania)

(58) Dans quels arrondissements on est aux sacs en plastique? (Forum «On m'a volé ma boîte verte »)

(59) Crise oblige, je suis aux carottes rapées [sic] Claude Léger à l'agence. Fini les restos entre cops. (Forum au féminin)

(60) Pour sauver le reste de ses besoins, une partie de plus en plus importante de la population est aux sandwichs. (Le Point, Les Français dévoreurs de sandwichs)

(61) Je ne cuisine pas beaucoup et en ce moment avec la chaleur j ai pas tres faim [sic] je suis aux tomates et salade c'est tout ce que je peux avaler. (Site Nous cherchons tous des recettes)

(62) en gros il y a 2 semaines "lourdes" à 200 environs avec lessive et stock divers et 2 plus "legeres" [sic] à 150; viande le soir puisque mon homme est aux sandwichs tous les midi. (Forum « Quel est votre budget de courses pour le mois ?») 
Je passe pas mal de temps à cuisiner, environ 1 h par repas. Mais en ce moment mon pauv'mari est aux pizzas. (Forum « Repas...galère »)

(64) Moi je suis aux tomates cerises (il ne m'en reste que 2 ! sniff sniff). (Forum au féminin)

(65) perso je suis aux chaussures quasi plates ou gros talons de $3 \mathrm{~cm}$... (Forum Doctissimo.fr)

(66) Je suis aux jupes droites et aux talons hauts au boulot... (Blog machine-arrière)

L'être humain situé abstraitement par rapport aux circonstances avec, parfois, l'inférence d'une certaine dépendance apparaît dans le frame des traitements et des prescriptions tels que (67-70) :

(67) Mâchoire cassée, on m'a opérée pour fixer ma machoire [sic], j’ai des plaquettes de métal...je suis aux liquides encore quelque temps...

(68) Depuis une semaine je suis aux morceaux et je mache [sic]. Mon seul soucis et [sic] que j'ai faim trois fois par jour...

(69) Mon rhume va mieux ; je suis aux poches de gel..

(70) Pour les chaussures, je suis aux chaussures médicales hélas depuis 1 ans 1/2 [sic] car les semelles orthopédiques que j'ai ne permettent rien d'autre hélas...

Il faut signaler, dans certains exemples de cette section, la co-présence d'indicateurs temporels (depuis, en ce moment, encore quelque temps) qui insèrent l'énoncé dans un déroulement, une évolution. Dimension temporelle qui devient plus explicite dans des énoncés du type 'Il en est à son troisième whisky', exemple du Robert, où l'ajout de en, renforce l'interprétation processuelle.

En effet, un scénario temporel semble s'établir dans ces énoncés pour évoquer des stades dans un déroulement socialement institué, par exemple dans la composition des repas associés aux heures de la journée (71), au rituel associé à la fin du repas (72), aux parcours habituels dans les aéroports (enregistrement des bagages, formalités, café, boissons au buffet) en (73).

Les croissants, c'est pour le matin...maintenant, on en est aux sandwichs !

(72) On en est aux alcools, l'ambiance devient un peu somnolence de bon aloi, la maison de Paul est double, deux pavillons séparés par le jardin.

(73) Pour faire un dernier salut à Bangkok, et pendant qu'on en est aux boissons, en voici une autre, chaude. Excellent latte, servi dans une buvette de chaîne du soi Thaniya.

Depuis la mort de papa, elle en est aux surgelés..

(75) Les distributeurs continuent à se diversifier : maintenant on en est aux pizzas fraîches.

Situant la cible par rapport au site dans le cadre d'un stade, une phase d'un déroulement, ces énoncés mettent en évidence l'ancrage temporel des repérages situationnels. En (74) on sent la dérive, à partir d'un moment précis (la mort de papa), par rapport aux habitudes passées (présupposées) ; en (75), on identifie une étape d'un développement commercial stratégique.

Un dernier exemple :

(76) Mais je fonctionne par phase...Il n'y a pas longtemps j'étais dans les jupes, puis je suis passée aux sacs, puis aux pantalons et là je suis aux chaussures, mais j'en suis à 3 paires en 1 mois. Donc je vais me calmer. [Forum «Votre péché mignon en fringues »]

Cet exemple est éloquent. Outre les repères temporels (Il n'y a pas longtemps, puis, puis, là) et la spatialisation du point de départ (dans les jupes), l'évolution est clairement signifiée par le verbe passer (avec l'inférence d'un chemin) et l'indication du trajet (jupes $>$ passer aux sacs $>$ aux pantalons $>$ être aux chaussures $>$ en être à 3 paires en 1 mois) avec, en fin de parcours, des résolutions voire des inférences d'ordre axiologique (mais...Donc je vais me calmer). Dans cet exemple, les objets prépositionnels fonctionnent comme les jalons de la trajectoire temporelle et notionnelle de l'acheteuse. Ici encore, la puissance évocatrice du verbe être participe de manière décisive à la construction du sens. 
Encore une remarque au sujet des énoncés précités. Ainsi que nous l'avons signalé pour les lieux (cf. 2.1 être à la cuisine $\rightarrow$ cuisiner, manger), certains SP avec des noms d'objet semblent aptes à évoquer plus d'un rapport. C'est le cas de aux liquides qui évoque le travail, la fonction en (32) (être aux liquides $\rightarrow$ travailler au rayon des liquides) et aussi un régime en (67) (être aux liquides $\rightarrow$ absorber des liquides). C'est aussi le cas de aux chaussures qui, avec plus ou moins de spécificité, induit une habitude vestimentaire en (65) (être aux chaussures plates $\rightarrow$ porter des chaussures plates) et une conduite d'achat en (76) (être aux chaussures $\rightarrow$ acheter des chaussures). D'où, au moins, trois conclusions. D'abord, le recouvrement des domaines dans notre classement: au lieu de dessiner des ensembles étanches et discrétisables, nous concevons des frontières floues et des intrications interdomaniales (espace-tempsnotion) variables selon les cas ; ensuite l'importance des ajustements contextuels pour définir le rapport pertinent - qui n'est pas prédéterminé par les propriétés ontologiques des entités dénotées - ; en dernier lieu, l'analyse de la construction du sens comme un processus dynamique avec des stabilisations transitoires selon un rapport praxéologique à la réalité intersubjective hautement dépendant des contraintes pragmatiques. Voilà qui nous conduit à ouvrir, pour les SP en à + nom d'objet, une dernière catégorie caractérisée par sa forte dépendance contextuelle.

\subsubsection{Objet $\rightarrow$ Interaction pertinente selon le contexte}

Cette catégorie regroupe des cas particuliers, résultat d'une connexion métonymique assurée selon des critères pragmatiques précis et variables selon le contexte. En effet, certains énoncés activent des inférences contingentes, tributaires des scénarios associés en vertu de notre connaissance du monde, sans que ces inférences supposent une lecture nécessairement et exclusivement localisatrice, télique, agentive, indiquant une habitude de consommation ou un stade dans une évolution. Ce dernier type de repérage tiendrait à une interprétation contextuelle adaptée à chaque cas selon des critères pragmatiques particuliers. Nous analyserons deux énoncés attestés :

Mon domaine quotidien c'est «aux casseroles derrière un fourneau », et quand j'ai un peu de temps « aux copeaux derrière un rabot». («Sondage CyberBricoleur » BHV)

Cet énoncé active une connexion particulière, liée au contexte. Ainsi, si aux casseroles renvoie à l'interprétation télique caractérisée en 3.3.2, aux copeaux semble induire un repérage moins bien défini : interprétable en termes d'activité associée (menuiserie) - et non pas de lieu (3.3.1) -, être aux copeaux n'est pas pour autant analysable en termes de rôle télique (les copeaux n'étant pas un instrument destiné à une activité), ni, à strictement parler, de rôle agentif (3.3.3) puisque, bien que la cible se trouve à l'origine du site (copeaux), il semble contre-intuitif de les considérer comme le fruit d'une activité intentionnelle de production, conception, confection (On n'est pas aux copeaux comme on est aux costumes). Il s'agirait plutôt d'un élément typique généré, certes, par le travail du bois mais contribuant à une indication indirecte de l'activité par l'évocation d'entités co-présentes, résultat naturel subsidiaire de l'action effectuée.

Le repérage situationnel décrit en 3.3.4 semblerait, à première vue, adéquat. Or cet emploi diffère des exemples étudiés comme repérage situationnel, généralement déterminés par un certain mode de consommation (et non pas 'conséquence', 'effet' ou 'résidu' de l'activité de la cible) ou même d'un stade dans une évolution (il y a certes habitude mais non pas parcours temporel / notionnel). C'est pourquoi, plutôt que de forcer une inclusion en 3.3.4, nous réservons à cet énoncé un traitement $a d$ hoc.

(78) On se lance, on se fait faire toutes les deux une french manucure (il faut bien essayer un jour lol) Et puis pendant que je suis aux ongles ${ }^{17}$ Sylvie fait un essai maquillage. Rien ne nous arrête, et hélène [sic] nous attendait gentiment (« Salon mariage Chantilly » Texte et photos)

L'interprétation de être aux ongles ne semble pas non plus réductible aux catégories précédentes : le SP ne semble pas orienté vers une interprétation locative mais plutôt vers une inférence d'activité, favorisée par l'environnement linguistique : être aux ongles reprend se faire faire une french manucure, le marqueur pendant renforce une interprétation en termes d'activité ; soulignons en outre que, ici, les ongles ne désignent pas métonymiquement le salon de beauté ni un secteur particulier de ce salon. Etre aux ongles n'admet pas non plus d'interprétation télique, les ongles n'étant pas un instrument pour 
l'effectuation d'une action. Le SP ne paraît pas non plus analysable en termes de rôle agentif puisque dans ce contexte la cible n'est pas attachée intentionnellement à la production ou conditionnement de l'entité désignée par le site (elle se fait faire une french manucure). Encore une fois, l'interprétation situationnelle, certes disponible du fait de sa souplesse, ne semble pas convenir au sens strict : il ne s'agit pas d'un repérage qualitatif dans le cadre d'un type de consommation / achat / utilisation de l'entité désignée par l'objet prépositionnel : je suis aux ongles ne semble pas paraphrasable par j'utilise / achète / mange des ongles (On n'est pas aux ongles comme on est aux piles rechargeables, aux sandwichs, aux chaussures, aux tomates cerise ou aux poches de gel). Autrement dit, le rapport métonymique instancié par ce syntagme prépositionnel joue sur des connexions pragmatiques particulières découlant du contexte qui justifient - nous semble-t-il - son inclusion dans une catégorie plus ouverte et plus adaptée aux aléas du discours.

On le voit, certains emplois semblent échapper à une taxinomie rigide (cela s'insinue en 3.3.4 et soustend l'existence de 3.3.5). L'interprétation de telles formulations est donc bel et bien contextuelle. Ainsi, plutôt que d'associer une interprétation fixe aux noms d'objet sur la base des entités dénotées, nous proposons une typologie qui prend en compte le contexte intervenant de manière dynamique et multidirectionnelle dans la construction du sens. En effet, le comportement sémantique et pragmatique des syntagmes prépositionnels s'avère contextuellement variable ; ainsi, si être aux casseroles suggère, dans la plupart des cas, l'action de cuisiner, des emplois moins prédictibles se présentent avec, par exemple, la séquence être aux pizzas : on pourrait dire qu'on est aux pizzas, dans une grande surface, lorsqu'on se trouve dans le rayon correspondant (simple localisation ou inférence d'activité quel que soit le rôle de la cible : chef de rayon, employé libre-service, client...), mais aussi lorsqu'on est simplement en train d'en préparer, d'en délivrer ou d'en consommer, activant une interprétation pragmatique en termes d'interaction pertinente, adaptée à chaque cas, interprétation variable à laquelle contribue le potentiel de la préposition à comme déclencheur d'inférences sur la base des connaissances partagées.

\section{Conclusion}

Après avoir passé en revue les travaux existants sur la localisation évoquée par les syntagmes en $a$ + nom d'objet précédé de l'article défini, nous avons réexaminé la question à partir d'énoncés attestés pris en contexte. Avec une perspective cognitive, notre analyse a rendu compte du rôle de la préposition $\grave{a}$ comme déclencheur d'inférences, de la dimension praxéologique des objets (les lexèmes les dénotant ont été définis comme des clés d'accès à des scénarios familiers) et de l'importance du contexte dans les connexions métonymiques à l'œuvre dans le discours selon des critères pragmatiques.

Certains aspects n'on pas été abordés dans ce travail ; cela ne veut pas dire pour autant qu'ils ont été exclus de nos considérations : nous pensons particulièrement au rôle conjoint de la préposition $\grave{a}$ et de l'article défini (traité, par exemple, dans Corblin (2011) et Aurnague (à paraître)), aux différences d'acceptabilité sémantico-pragmatique entre le singulier et le pluriel, à la variété des facettes des lexèmes (Cruse, 1986) intégrant les syntagmes localisateurs ainsi qu'à l'intrication, dans la pratique discursive, des domaines spatial, temporel et notionnel. À ce sujet, la typologie proposée en fin de parcours revêt les caractéristiques d'une construction souple et continue qui tient compte des liens pragmatiques omniprésents dans les pratiques langagières.

Ce travail appellera bien sûr des prolongements, des ajustements, des approfondissements...

\section{Références bibliographiques}

Aurnague, M. (2004). Les structures de l'espace linguistique : regards croisés sur quelques constructions spatiales du basque et du français. Leuven/Paris : Peeters. 
Aurnague, M. (2009). À cet endroit vs. dans un tel endroit : ce que à nous dit d'endroit et vice-versa. Langages, 173, 34-53.

Aurnague, M. (2010). Places-repère, localisation et routines : lorsque l'analyse du nom place rejoint celle de la préposition à. CORELA - Espace, Préposition, Cognition | Numéros thématiques. [En ligne] Publié en ligne le 31 mai 2010. URL : http://corela.edel.univ-poitiers.fr/index.php?id=919

Aurnague, M. (à paraître). Quand la routine s'installe : remarques sur les emplois de à de type 'routine sociale'.

Borillo, A. (1999). L'espace et son expression en français. Paris : Ophrys.

Borillo, A. (2001). La détermination et la préposition de lieu à en français. Lingvisticae Investigationes Supplementa, 23. Amsterdam : John Benjamins, 85-99.

Brown, G. (1965). Social Psychology. New York : Free Press.

Bruneau, F., Bruneau, C. (1956 [1933]). Précis de grammaire historique de la langue française. Paris : Masson et Cie.

Cadiot, P. (1997a). Les paramètres de la notion de préposition incolore. Faits de Langues, 9, 127-134.

Cadiot, P. (1997b). Les prépositions abstraites en français. Paris : Armand Colin.

Cadiot, P., Nemo, F. (1997a). Pour une sémiogenèse du nom. Langue Française, 113, 24-34.

Cadiot, P., Nemo, F. (1997b). Propriétés extrinsèques en sémantique lexicale. Journal of French Language Studies, 7-2, 127-146.

Corblin, F. (2011). Locus et telos: aller à l'école, être à la plage. Article soumis. Disponible sur: $\underline{\mathrm{http}: / / \text { fcorblin.free.fr/textes/articletempsespace.pdf }}$

Cruse, D. A. (1986). Lexical Semantics. Cambridge: Cambridge University Press.

Fillmore, C. (1982). Frame semantics. Linguistics in the Morning Calm. Linguistic Society of Korea (ed.), Seoul: Hanhin, 111-137.

Franckel, J.-J. et Lebaud, D. (1992). Lexique et opérations. Le lit de l'arbitraire. La théorie d'Antoine Culioli. Ouvertures et incidences. Paris : Ophrys, 89-105.

Hernández, P. (2007). Effets de focalisation et de cadrage dans la mise en images de la localisation et de la configuration. Eléments pour une sémantique des prépositions spatiales en français et en espagnol. Prépositions françaises à - en - dans - chez - sur. Prépositions espagnoles a - en - sobre. Thèse de doctorat. Université de Rouen.

Hernández, P. (2010). La préposition française à et les sites scénarisés. In Canello, M., Buscaglia, M. (eds). Actas de las XV Sesiones para docentes e Investigadores de Francés Lengua Extranjera "1810- 2010: del francés del Iluminismo al francés de hoy”. Rosario : Laborde Libros Editor (support électronique sans pagination).

Huyghe, R. (2009). Les noms généraux d'espace en français. Enquête linguistique sur la notion de lieu. Bruxelles : De Boeck - Duculot.

Jackendoff, R. (1983). Semantics and Cognition. Cambridge: M.I.T. Press.

Janet, P. (1935). Les débuts de l'intelligence. Paris: Flammarion. Version numérique réalisée par Jean-Marie Tremblay le 11 mai 2003 à Chicoutimi, Québec à partir de l'article de Pierre Janet (1932). Disponible sur : http://classiques.uqac.ca/classiques/janet pierre/debut de intelligence/debut intelligence.html

Katz, E. (2002). Systématique de la triade spatiale à, en, dans. Travaux de linguistique, 44, 35-49. Disponible sur : http://www.cairn.info/revue-travaux-de-linguistique-2002-1-page35.htm

Lakoff, G. (1987). Women, Fire and Dangerous Things. What Categories Reveal about the Mind. Chicago/London: University of Chicago Press.

Langacker, R. (1987). Foundations of Cognitive Grammar. Stanford: Stanford University Press.

Le Robert Electronique (1994). Dictionnaires Le Robert.

Melis, L. (2003). La préposition en français. Paris : Ophrys. 
Pustejovsky, J. (1995). The generative lexicon. Cambridge : M.I.T Press.

Radden, G., Kövecses, Z. (1999). Towards a theory of metonymy. Panther, K. \& Radden, G. (eds). Metonymy in language and thought. Amsterdam - Philadelphia: John Benjamins, 17-59.

Siblot, P. (1997). Nomination et production du sens : le praxème. Langages, 127, 38-55.

Spang-Hanssen, E. (1963). Les prépositions incolores du français moderne. Copenhague : G.E.C. Gads Forlag.

Trésor de la langue française informatisé. Disponible sur : $\mathrm{http}: / /$ atilf.atilf.fr/tlfi.htm

Vandeloise, C. (1986). L'espace en français. Paris : Éditions du Seuil.

Vandeloise, C. (1988). Les usages spatiaux statiques de la préposition à. Cahiers de Lexicologie, 53, 119-148.

Vandeloise, C. (1990). Les frontières entre les prépositions sur et à. Cahiers de Grammaire, 15, 159-184.

Von Wartburg, W., Zumthor, P. (1973 [1947]). Précis de syntaxe du français contemporain. Suisse : Francke Berne.

\footnotetext{
${ }^{1}$ Cette communication a bénéficié des remarques d'un relecteur anonyme. Nous tenons à lui exprimer notre plus vive reconnaissance.

${ }^{2}$ Nous empruntons à Vandeloise (1986:34) les termes cible (entité à localiser) et site (point de repère).

${ }^{3}$ A ce propos, il ne faut pas négliger le fait que la préposition à peut introduire des syntagmes avec des noms référant à des activités, par exemple Baudoin est à une réunion (Vandeloise, 1988: 130) ou Je les ai vus à la réunion du bureau (Melis, 2003 : 65). Le marqueur intervient aussi dans des périphrases du type être $\grave{a}+$ Infinitif avec le sens de 'être en train de' : "Tous deux devaient être à causer sous le hangar, lorsque lui s'était avancé pou appeler le souschef. » E. Zola, La Bête humaine, 1890, p. 59, cité par le Trésor de la Langue Française informatisé.
}

${ }^{4}$ Nous n'aborderons pas ici la question des Noms de Localisation Interne (Borillo 1999), désignés par Vandeloise (1988) comme 'localisations par rapport aux parties d'un objet'. Nous n'entamerons pas non plus de discussion sur la nature du défini complément (cf. Corblin, 2011).

${ }^{5}$ À moins, bien sûr, qu'il ne s'agisse d'une école pour des chiens. C'est le cas d'une école pour les chiens guides d'aveugles. Énoncé attesté : « [...] le chien est à l'école dans la semaine et revient dans sa famille d'accueil le weekend. Quand le chien a fini sa formation, un éducateur le remet à son futur maître ». On trouve ici une interaction pertinente entre la cible (le chien) et le site (l'institution éducative). C'est l'une des seules quatre occurrences de ce SP sur le web, dont deux à caractère métalinguistique.

${ }^{6}$ L'auteur cite le cas des 'situations de jeu' où des objets inaptes à la localisation (chaise, tabouret) peuvent être interprétés comme des lieux servant de jalon : Maintenant, je suis à la chaise / au tabouret! (2009: 37 note 3)

${ }^{7}$ Il convient à ce stade de rappeler deux des quatre rôles prévus par la Structure de Qualia (Pustejovsky, 1995) : rôle télique (but et fonction de l'objet, par exemple livre $\rightarrow$ lire) et rôle agentif (origine de l'objet, conditions d'apparition, par exemple livre $\rightarrow$ écrire)

${ }^{8}$ Pour tous les énoncés cités, nous transcrivons les formulations sous leur forme d'origine sur Internet.

${ }^{9}$ Nous n'analyserons pas ici la question du choix entre singulier et pluriel ni celle de la fixation de l'une des deux formes. Parfois, les deux formes sont attestées, par exemple, être au fourneau / aux fourneaux, être à la cuisine / aux cuisines - cette dernière expression associée, en général, au domaine de la restauration. Dans d'autres cas, l'indication du nombre s'accompagne d'une nuance distinctive qui fixe l'un des deux emplois : à la casserole, au singulier, désigne un mode de cuisson ("c'est à la casserole et non au micro-ondes »), le pluriel aux casseroles renvoie à l'activité (« Jérôme est aux casseroles et Raphaële s'occupe du chérubin »). De manière générale, l'emploi du pluriel, qui semble se prêter plus aisément aux inductions, impose moins de contraintes que le choix du singulier. Cette question mériterait de plus amples développements qui dépassent le cadre de ce travail.

${ }^{10}$ Bien entendu, des cas marginaux sont toujours possibles : on peut 'être au lit dans le salon' ou 'être à table dans le jardin' où l'inférence d'activité typique (dormir/ se reposer, manger) associée au site se trouve située dans un espace particulier (salon, jardin) marqué par le fait de sa propre discordance avec le décor attendu (chambre à coucher, salle à manger). Or la connexion conceptuelle entre l'activité suscitée par l'entité (lit $\rightarrow$ dormir, table $\rightarrow$ manger) et le cadre habituel (chambre, salle à manger) n'est pas interrompue pour autant et demeure disponible, prête à être 
convoquée par l'objet en question. Ainsi, il serait parfaitement possible d'entendre et d'accepter dans le discours des formulations telles que «Le salon c'est ma chambre à coucher, j'y ai installé mon lit » ou «On va se mettre à table. Je vous prie de me suivre dans la salle à manger » avec un geste d'ostension vers le jardin où la table a été dressée. Car, indépendamment de la forme linguistique choisie, notre rapport praxéologique aux lieux / entités rend acceptables ces formulations en vertu de notre expérience (propriétés extrinsèques selon Cadiot et Nemo, 1997a, 1997b) et des scénarios institués.

${ }^{11}$ Selon le classement de Aurnague (2009: 36), le terme cuisine désignerait une entité mixte (cf. 2.2.3). Nous le prenons ici comme un nom de lieu - l'auteur signale d'ailleurs l'existence de cas de recatégorisation (coercition de type).

${ }^{12}$ Le terme place correspond, dans ces énoncés, à ce que Vandeloise $(1999,2001)$ dénomme place-repère découlant du concept d'ordre établi, par exemple dans Un bar n'est pas la place d'une jeune fille où se font jour des conventions d'ordre social. Suivant Vandeloise (2001), Huyghe (2009) signale la 'valeur normative' de place (« là où $\mathrm{X}$ doit se trouver ») qui conduit à une certaine institution des places dans un univers donné. Aurnague (2010) analyse des énoncés tels que La place d'un produit surgelé est au congélateur, La place d'un pianiste est au piano, La place d'un marmiton est au fourneau. Sous-tendus par des routines associées à des sites intégrés (au congélateur, au piano, au fourneau), ces énoncés paraissent mettre en jeu une certaine forme d'activité, souligne le linguiste, plutôt qu'une simple localisation spatiale.

${ }^{13}$ Un détail : selon le Trésor de la langue française informatisé, la plonge désigne d'abord l'activité (lavage de la vaisselle dans un restaurant, une collectivité) et par métonymie le lieu ou la cuve où se fait cette opération. On voit donc ici un transfert métonymique activité $\rightarrow$ lieu.

${ }^{14}$ De même que les objets, les activités permettent d'identifier les lieux où elles se déroulent habituellement : ainsi, dans un atelier ou entreprise, et selon les métiers, Paul est au montage / Pierre est au laquage / Marie est à l'assemblage. Un exemple: "Je sors et si on me cherche, je suis au maquillage ». A remarquer la productivité du lexème attestée dans le dictionnaire (maquillage $=$ action $>$ résultat $>$ produits de beauté) ou activée pragmatiquement (lieu).

${ }^{15}$ Dans cet énoncé, l'emploi du singulier semble 'démonter' la métonymie, fixée au pluriel, être aux casseroles = cuisiner. Ce singulier 'contingent' renvoie le lexème à son rôle dénotatif et fait un clin d'œil descriptif sur la situation.

${ }^{16}$ Pour une question d'extension, nous n'analyserons pas en détail cette différence sémantique - valable pour les rapports métonymiques étudiés (3.3.2 - 3.3.5) - que nous réservons pour de futurs développements.

${ }^{17}$ Nous n'analyserons pas ici la relation partie-tout (corps $>$ membres $>$ main $>$ ongles) et nous traiterons le terme ongles comme désignant un 'objet' i.e. ce qui n'est pas un lieu (Cf. 2.2.3). 